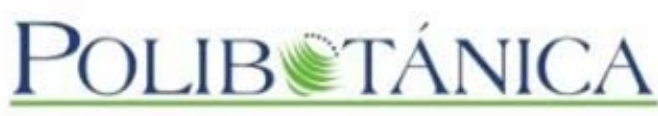

\title{
Polibotánica
}

ISSN electrónico: 2395-9525

polibotanica@gmail.com

Instituto Politécnico Nacional

México

http://www.polibotanica.mx

\section{VARIABILIDAD MORFOLÓGICA DE CHICOZAPOTE DE NAYARIT, MÉXICO.}

\section{MORFOLOGIC VARIABILITY OF CHICOZAPOTE FROM NAYARIT, MÉXICO.}

Arrieta-Ramos, B.G., A. Villegas-Monter, P.G. Ruelas-Hernández, C.R. Juárez-Rossete y K.J. Partida-Lucian.

VARIABILIDAD MORFOLÓGICA DE CHICOZAPOTE DE NAYARIT, MÉXICO. MORFOLOGIC VARIABILITY OF CHICOZAPOTE FROM NAYARIT, MÉXICO.

POLIBETANICA

Instituto Politécnico Nacional
Núm. 49: 93-106 México. Enero 2020

DOI: $10.18387 /$ polibotanica.49.6

(c) (i) Este es un artículo de acceso abierto bajo la licencia Creative Commons 4.0 Atribución-No Comercial (CC BY-NC 4.0 Internacional). 
VARIABILIDAD MORFOLÓGICA DE CHICOZAPOTE DE NAYARIT, MÉXICO

\section{MORFOLOGIC VARIABILITY OF CHICOZAPOTE FROM NAYARIT, MÉXICO}

Arrieta-Ramos, B.G.,

A. Villegas-Monter,

P.G. Ruelas-Hernández,

C.R. Juárez-Rossete

y K.J. Partida-Lucian.

VARIABILIDAD

MORFOLÓGICA DE

CHICOZAPOTE DE

NAYARIT, MÉXICO.

MORFOLOGIC

VARIABILITY OF

CHICOZAPOTE FROM

NAYARIT, MÉXICO.

\section{POLIBETÁNICA}

Instituto Politécnico Nacional

Núm. 49: 93-106. Enero 2020

DOI:

10.18387/polibotanica.49.6
B.G. Arrieta Ramos / g-arrieta@ hotmail.com Unidad Académica de Agricultura, Universidad Autónoma de Nayarit, Km 9.5 de la Carretera Tepic-Compostela, Xalisco, Nayarit, Méx. CP 63780. Tel 01 (31) 12110128

A. Villegas Monter Colegio de Posgraduados Campus Montecillo, Km. 36.5, México 136 5, Montecillo, Montecillo, Méx. CP 56230.

P.G. Ruelas Hernández C.R. Juárez Rossete K.J. Partida Lucian Unidad Académica de Agricultura, Universidad Autónoma de Nayarit, Km 9.5 de la Carretera Tepic-Compostela, Xalisco, Nayarit, Méx.

RESUMEN: El chicozapote es una especie introducida en el estado de Nayarit y, dado que, la diseminación fuera de su región de origen contribuye a la diversificación de las especies, el objetivo de esta investigación fue conocer la variabilidad morfológica en árboles de chicozapote de Nayarit, México, e identificar materiales sobresalientes para producción de fruta. Se realizaron recorridos de campo, se ubicaron y caracterizaron 55 árboles. Se tomaron variables morfológicas y agronómicas. Se realizó estadística descriptiva, análisis de correlación, componentes principales y análisis de conglomerados jerárquicos. Se encontró variabilidad entre los árboles de chicozapote. En el análisis de componentes principales los tres primeros componentes explicaron el $79.13 \%$ de la variabilidad morfológica total, las variables que los determinaron fueron: diámetro ecuatorial y longitudinal del fruto, peso del fruto y peso de la pulpa, ancho de la hoja y peso de la semilla. El análisis de conglomerados diferenció cinco grupos; y, al interior de los grupos, se presentó variabilidad que permitió diferenciar subgrupos. Los grupos no se separaron por municipio en el dendograma. Existe variabilidad morfológica entre los materiales de chicozapote de Nayarit. Algunos de los materiales presentan características adecuadas para producción de fruta: vida postcosecha larga, no poseer semillas y cáscara gruesa, que pueden ser evaluados por sus características organolépticas. Los árboles productores de frutos partenocárpicos tienen el potencial de ser utilizados para estudios de biología reproductiva de chicozapote

Palabras clave: Manilkara zapota, recursos fitogenéticos, caracterización.

ABSTRACT: The chicozapote is an introduced species in the state of Nayarit, and given that, the dissemination of species outside of their originary region contributes to species diversification, this research was conducted with the purpose of knowing the morphologic variability of chicozapote trees of Nayarit, México and identifying outstanding materials for fruit production. Field trips around the State were carried out, and 55 trees were located and characterized. Morphologic and agronomic variables were registered. The data analysis used descriptive statistics, correlation analysis, principal components analysis and hierarchical cluster analysis. Morphological variability is present in chicozapote materials of Nayarit. In the analysis of principal 
components, the first three components explained $79.13 \%$ of the total morphological variability, and the variables that determined it were: equatorial and longitudinal fruit diameter, weight of fruit and pulp, width of leaf and weight of seed. The cluster analysis differentiated five groups, and inside of the groups, variability that allowed differentiating subgroups was present. The groups were not separated by municipality in the dendrogram. Morphological variability exists among the chicozapote materials of Nayarit. Some of the materials identified have potential to be used for fruit production, thick shell, long post-harvest life and seedless fruits that could be evaluated for organoleptic properties. The trees that produce parthenocarpic fruit could be used for studies about the reproductive biology of chicozapote.

Key words: Manilkara zapota, fitogenetic resources, characterization.

\section{INTRODUCCIÓN}

El chicozapote, es uno de los recursos fitogenéticos de México, ya que es originario del sudeste del País y América Central, región en la que una vez, hubo enormes poblaciones de esta especie (Brito \& Narain, 2002). Se dice que fue introducido en Filipinas en tiempos de la colonia, de ahí se extendió hasta Asia y se hizo popular en India y Malasia (Aldrete \& Ramírez, 2005). Vázquez, Batis, Alcocer, Gual y Sánchez (1999) consideraban que los frutos más selectos, se encuentran en Tailandia de donde se exportaban a Europa. Esta especie se cultiva en muchos países tropicales por el fruto, látex y madera.

En México, el árbol del chicozapote presenta importancia a nivel económico por la producción de chicle a partir del látex. De acuerdo con la Comisión Nacional de Áreas Naturales Protegidas (CONAP, 2016) en la reserva de la biosfera de Calakmul, Campeche, el látex se extrae del árbol de manera sustentable, desde hace más de 20 años por un grupo de 125 productores, que producen 10 toneladas de resina de chicle y generan de 4 a 6 millones de dólares al año. Sin embargo, para un manejo adecuado, se requiere que a los árboles se les deje descansar por un periodo de tres años, lo que limita la capacidad de producción de estas reservas naturales.

A pesar de su importancia, no existe suficiente información sobre esta especie que permita generar programas apropiados para su conservación, mejoramiento y manejo (González, García \& Guntin, 2012).

Actualmente, el chicozapote se distribuye en México, en el área del Pacífico, desde Chiapas hasta Nayarit, donde fue introducido (Villegas, 2012). La importancia de las introducciones radica en que pueden ser evaluadas como reserva de diversidad, ya que uno de los factores que contribuyen a la diversificación, es la diseminación de las especies fuera de su región de origen, debido a que las plantas se adaptan a las condiciones en que se desarrollan (Azurdia, 2006).

Los estudios sobre variabilidad son útiles para la conservación y manejo racional de las especies y la conservación de los recursos fitogenéticos, la sustentabilidad y la productividad (Piñero, Caballero, Cabrera, Canteros \& Casas, 2008). La variabilidad es una medida de la tendencia de los genotipos de una población a diferenciarse y está determinada por la variación de las características morfológicas que son poco afectadas por el ambiente y puede ser caracterizada (Osawaru, Ogwu \& Aiwansoba, 2015). Además, una manera de conservar es valorizando los recursos, lo que requiere la caracterización y evaluación para realizar el desarrollo de germoplasma específico (Paredes, Becerra, Condón, Germán \& Barbieri, 2006).

Los estudios sobre caracterización morfológica de chicozapote son limitados. Gazel, 2002 realizó la caracterización de 13 árboles de chicozapote que se encontraban en el banco de germoplasma del CATIE (Centro Agronómico Tropical de Investigación y Enseñanza) en Costa Rica. En esta colección se encontraban materiales de México y Estados Unidos entre otros. Se encontró que se formaron tres grupos, con cuatro, seis y tres plantas, respectivamente. En México, Aguilar, Torres, Corona, Juárez \& Villanueva (2018), realizaron la caracterización de 
árboles provenientes de dos regiones, el centro de Veracruz y el estado de Morelos. Encontraron que las variables sobresalientes para caracterizar al material de chicozapote evaluado fueron el ancho de hoja, flores por racimo, ancho de fruto, longitud del pedúnculo del fruto y peso del fruto. En ése trabajo, el análisis de conglomerados formo cuatro grupos principales, en el que los criollos del estado de Morelos eran poco semejantes al resto de los grupos de la región centro del Estado de Veracruz, por lo que consideraron que tienen un origen diferente.

También se ha realizado la caracterización de chicozapote con marcadores moleculares SSR en 20 árboles adultos de diferentes regiones fisiográficas del estado de Veracruz. Se encontró que el número de alelos fue alto (media de 11.85); la heterocigosidad fue baja (media de 0.154) y el análisis pudo distinguir dos grupos, uno con los materiales del norte del estado, el otro con subgrupos, del sur y centro del estado (González, García \& Guntin, 2012). En contraste, Heaton, Whitkus \& Gómez (2003) utilizando RAPDs no lograron distinguir dos poblaciones morfológicamente distintas provenientes de la Península de Yucatán, árboles de bosque, altos rectos y poblaciones de pantano de crecimiento corto, con forma arbustiva y lo atribuyeron a que el flujo de genes pudo haber impedido la acumulación de marcadores neutros o a que las diferencias morfológicas son debidas a una respuesta plástica al hábitat.

Existen también trabajos de caracterización postcosecha de frutos y se han estudiado parámetros de maduración, considerando sólo una variedad o frutos provenientes de un grupo de árboles (Bautista, Arévalo, Veloz \& Martínez, 2005; Brito \& Narain, 2002; Moo, Estrada, Estrada, Cuevas \& Sauri, 2013).

Aunque en México el chicozapote se utiliza principalmente para la extracción del látex, el fruto es apreciado en muchas partes del mundo, sin embargo, su comercialización es limitada debido al desarrollo escaso de la especie. Debido a lo anterior, este trabajo tuvo el objetivo de evaluar la variabilidad morfológica e identificar materiales con características para producción de fruta, en árboles de chicozapote en Nayarit que contribuyan al conocimiento, valorización y conservación de la especie.

\section{MATERIAL Y MÉTODOS}

\section{Área de estudio}

Se realizaron recorridos de campo para ubicar los árboles de chicozapote, en los municipios de San Blas, Santiago Ixcuintla, Tecuala, Compostela, Amatlán de Cañas, Ahuacatlán y Tuxpan, Nayarit.

\section{Material vegetal}

El material consistió en 55 árboles de chicozapote, provenientes de semilla, en producción, los cuales se ubicaron con un GPS Vista HCX (Garmín, Garmin Inc., Taiwan).

\section{Muestreo}

En cada árbol, se recolectaron cuatro muestras de brotes con hojas maduras, orientados a cada uno de los puntos cardinales. Los brotes fueron colocados en bolsas y etiquetados con el número de árbol. También se recolectaron muestras de 20 frutos en madurez físiológica, para ser llevados a la Unidad Académica de Agricultura, de la Universidad Autónoma de Nayarit, donde fueron caracterizados en madurez de consumo.

\section{Variables}

Se consideraron 13 variables: largo y ancho de la hoja, longitud del pedúnculo, peso total del fruto, peso de pulpa, semilla y cáscara, grosor de cáscara, número y peso de semillas, largo, ancho y grosor de semilla. En cada uno de los cuatro brotes tomados de cada árbol, se contaron las hojas y se seleccionaron 10 de tamaño medio, se midió: el largo desde la base hasta el ápice 
de la hoja, el ancho, en la parte media de la hoja, y la longitud del pedúnculo. El largo del fruto se midió desde la base del pedúnculo hasta el ápice; y el ancho, en la parte media del fruto. Se registró el peso total del fruto, enseguida se separó en pulpa, semilla y cáscara para obtener el peso de cada variable. El grosor de cáscara: se midió tomando dos lecturas por fruto, cada una en los extremos de la parte media del fruto. Se contó el número de semillas; enseguida se midió el largo, ancho y grosor de semilla de cada una de las semillas en los frutos. Para las variables de peso, se utilizó una balanza digital marca OHAUS modelo TS1205 (Corporation, N.J); y para las de longitud, se utilizó un vernier digital marca Truper (Truper Inc., México). Se registraron, además, las fechas de cosecha y los días de madurez fisiológica a madurez de consumo de las recolectas, estos datos no se utilizaron en el análisis estadístico.

\section{Análisis estadístico}

Para el análisis de las variables se obtuvo la matriz de correlación entre caracteres estandarizados y con la media de los valores obtenidos para cada variable, análisis de componentes principales y análisis de conglomerados jerárquicos mediante UPGMA (Unweighted Group Pair Method with Arithmetic Mean) utilizando la distancia euclidiana como medida de similitud. Los datos se procesaron con el Programa SAS® 9.2 (Statistical Analisis System, SAS Institute Inc.) y estadística descriptiva para caracterizar los grupos.

\section{RESUltados}

\section{Ubicación de los árboles}

La ubicación de los árboles se muestra en el cuadro 1. En el caso de Tuxpan, sólo se encontró un árbol. La altitud a la que se localizaron los árboles fueron desde los 4 hasta $1005 \mathrm{~m}$.

Los resultados de la caracterización de la hoja se muestran en el cuadro 2. Los mayores rangos en la longitud y ancho de la hoja y longitud del peciolo se presentaron en el municipio de Santiago, y los menores en Ahuacatlán.

En el cuadro 3 se presentan los valores promedio máximos y mínimos del peso de fruto y sus partes de las recolectas.

Los valores promedio mínimos y máximos de las características de fruto se presentan en el cuadro 4.

Cuadro 1. Ubicación geográfica y municipio de procedencia de los árboles de chicozapote caracterizados en Nayarit.

\begin{tabular}{|c|c|c|c|c|c|c|c|}
\hline Recolecta & Latitud & Longitud & $\begin{array}{l}\text { Altitud } \\
\text { (m) }\end{array}$ & Recolecta & Latitud & Longitud & $\begin{array}{l}\text { Altitud } \\
\text { (m) }\end{array}$ \\
\hline Ahuacatlán & & & & San Blas & & & \\
\hline 1 & $21^{\circ} 02^{\prime} 58.7^{\prime \prime}$ & $104^{\circ} 28^{\prime} 40.9^{\prime \prime}$ & 999 & 29 & $21^{\circ} 41^{\prime} 23.3^{\prime \prime}$ & $105^{\circ} 18^{\prime} 25.4^{\prime \prime}$ & 22 \\
\hline 2 & $21^{\circ} 02^{\prime} 58.7^{\prime \prime}$ & $104^{\circ} 28^{\prime} 40.9^{\prime \prime}$ & 999 & 30 & $21^{\circ} 41^{\prime} 17.9^{\prime \prime}$ & $105^{\circ} 18^{\prime} 24.6^{\prime \prime}$ & 21 \\
\hline 3 & $21^{\circ} 02^{\prime} 54.1^{\prime \prime}$ & $104^{\circ} 28^{\prime} 42.0^{\prime \prime}$ & 1005 & 31 & $21^{\circ} 40^{\prime} 34.5^{\prime \prime}$ & $105^{\circ} 19^{\prime} 34.5^{\prime \prime}$ & 21 \\
\hline 4 & $21^{\circ} 02^{\prime} 55.7^{\prime \prime}$ & $104^{\circ} 28^{\prime} 49.1^{\prime \prime}$ & 1002 & 32 & $21^{\circ} 40^{\prime} 43.3^{\prime \prime}$ & $105^{\circ} 19^{\prime} 37.7^{\prime \prime}$ & 4 \\
\hline \multicolumn{4}{|c|}{ Amatlán de Cañas } & 33 & $21^{\circ} 32^{\prime} 34.4^{\prime \prime}$ & $105^{\circ} 16^{\prime} 59.3^{\prime \prime}$ & 10 \\
\hline 5 & $20^{\circ} 49^{\prime} 09.5^{\prime \prime}$ & $104^{\circ} 23^{\prime} 07.3^{\prime \prime}$ & 768 & 34 & $21^{\circ} 39^{\prime} 44.8^{\prime \prime}$ & $105^{\circ} 22^{\prime} 04.2^{\prime \prime}$ & 12 \\
\hline 6 & $20^{\circ} 48^{\prime} 42.3^{\prime \prime}$ & $104^{\circ} 23^{\prime} 55.9^{\prime \prime}$ & 771 & 35 & $21^{\circ} 39^{\prime} 51.8^{\prime \prime}$ & $105^{\circ} 21^{\prime} 39.3^{\prime \prime}$ & 13 \\
\hline 7 & $20^{\circ} 48^{\prime} 42.8^{\prime \prime}$ & $104^{\circ} 23^{\prime} 56.2^{\prime \prime}$ & 770 & 36 & $21^{\circ} 39^{\prime} 47.4^{\prime \prime}$ & $105^{\circ} 21^{\prime} 46.2^{\prime \prime}$ & 4 \\
\hline
\end{tabular}




\begin{tabular}{|c|c|c|c|c|c|c|c|}
\hline Recolecta & Latitud & Longitud & $\begin{array}{l}\text { Altitud } \\
\text { (m) }\end{array}$ & Recolecta & Latitud & Longitud & $\begin{array}{l}\text { Altitud } \\
\text { (m) }\end{array}$ \\
\hline 8 & $20^{\circ} 48^{\prime} 46.7^{\prime \prime}$ & $104^{\circ} 23^{\prime} 57.1^{\prime \prime}$ & 774 & 37 & $21^{\circ} 40^{\prime} 44.3^{\prime \prime}$ & $105^{\circ} 21^{\prime} 11.2^{\prime \prime}$ & 13 \\
\hline 9 & $20^{\circ} 48^{\prime} 25.7^{\prime \prime}$ & $104^{\circ} 24^{\prime} 24.2^{\prime \prime}$ & 758 & 38 & $21^{\circ} 40^{\prime} 19.8^{\prime \prime}$ & $105^{\circ} 19^{\prime} 37.8^{\prime \prime}$ & 12 \\
\hline 10 & $20^{\circ} 51^{\prime} 31.8^{\prime \prime}$ & $104^{\circ} 25^{\prime} 51.3^{\prime \prime}$ & 930 & Santiago & & & \\
\hline 11 & $20^{\circ} 48^{\prime} 38.4^{\prime \prime}$ & $104^{\circ} 23^{\prime} 59.1^{\prime \prime}$ & 774 & 39 & $21^{\circ} 42^{\prime} 16.7^{\prime \prime}$ & $105^{\circ} 07^{\prime} 46.9^{\prime \prime}$ & 44 \\
\hline 12 & $20^{\circ} 48^{\prime} 39.3^{\prime \prime}$ & $104^{\circ} 23^{\prime} 58.9^{\prime \prime}$ & 774 & 40 & $21^{\circ} 42^{\prime} 17.5^{\prime \prime}$ & $105^{\circ} 07^{\prime} 47.6^{\prime \prime}$ & 45 \\
\hline 13 & $20^{\circ} 48^{\prime} 39.3^{\prime \prime}$ & $104^{\circ} 23^{\prime} 59.1^{\prime \prime}$ & 774 & 41 & $21^{\circ} 46^{\prime} 33.2^{\prime \prime}$ & $105^{\circ} 10^{\prime} 28.4^{\prime \prime}$ & 22 \\
\hline Compostela & & & & 42 & $21^{\circ} 48^{\prime} 49.3^{\prime \prime}$ & $105^{\circ} 11^{\prime} 38.4^{\prime \prime}$ & 17 \\
\hline 14 & $21^{\circ} 03^{\prime} 25.7^{\prime \prime}$ & $105^{\circ} 14^{\prime} 08.1^{\prime \prime}$ & 5 & 43 & $21^{\circ} 48^{\prime} 52.4^{\prime \prime}$ & $105^{\circ} 12^{\prime} 45.8^{\prime \prime}$ & 16 \\
\hline 15 & $21^{\circ} 02^{\prime} 30.3^{\prime \prime}$ & $105^{\circ} 12^{\prime} 39.6^{\prime \prime}$ & 16 & 44 & $21^{\circ} 48^{\prime} 06.1^{\prime \prime}$ & $105^{\circ} 12^{\prime} 21.7^{\prime \prime}$ & 17 \\
\hline 16 & $21^{\circ} 03^{\prime} 58.9^{\prime \prime}$ & $105^{\circ} 13^{\prime} 38.7^{\prime \prime}$ & 14 & 45 & $21^{\circ} 48^{\prime} 06.0^{\prime \prime}$ & $105^{\circ} 12^{\prime} 22.7^{\prime \prime}$ & 17 \\
\hline 17 & $21^{\circ} 11^{\prime} 06.6^{\prime \prime}$ & $105^{\circ} 08^{\prime} 14.9^{\prime \prime}$ & 30 & 46 & $21^{\circ} 48^{\prime} 04.8^{\prime \prime}$ & $105^{\circ} 12^{\prime} 07.0^{\prime \prime}$ & 21 \\
\hline 18 & $21^{\circ} 04^{\prime} 45.5^{\prime \prime}$ & $105^{\circ} 12^{\prime} 00.4^{\prime \prime}$ & 45 & 47 & $21^{\circ} 46^{\prime} 35.4^{\prime \prime}$ & $105^{\circ} 10^{\prime} 26.6^{\prime \prime}$ & 19 \\
\hline 19 & $21^{\circ} 06^{\prime} 44.4^{\prime \prime}$ & $105^{\circ} 12^{\prime} 01.1^{\prime \prime}$ & 42 & Tecuala & & & \\
\hline 20 & $21^{\circ} 06^{\prime} 04.5^{\prime \prime}$ & $105^{\circ} 12^{\prime} 01.3^{\prime \prime}$ & 13 & 48 & $22^{\circ} 23^{\prime} 45.7^{\prime \prime}$ & $105^{\circ} 27^{\prime} 18.8^{\prime \prime}$ & 5 \\
\hline 21 & $21^{\circ} 06^{\prime} 05.6^{\prime \prime}$ & $105^{\circ} 12^{\prime} 01.6^{\prime \prime}$ & 14 & 48 & $22^{\circ} 26^{\prime} 48.4^{\prime \prime}$ & $105^{\circ} 24^{\prime} 34.9^{\prime \prime}$ & 42 \\
\hline 22 & $21^{\circ} 06^{\prime} 07.6^{\prime \prime}$ & $105^{\circ} 12^{\prime} 01.8^{\prime \prime}$ & 16 & 50 & $22^{\circ} 26^{\prime} 48.6^{\prime \prime}$ & $105^{\circ} 24^{\prime} 34.9^{\prime \prime}$ & 41 \\
\hline 23 & $21^{\circ} 04^{\prime} 46.3^{\prime \prime}$ & $105^{\circ} 09^{\prime} 08.0^{\prime \prime}$ & 248 & 51 & $22^{\circ} 26^{\prime} 47.1^{\prime \prime}$ & $105^{\circ} 24^{\prime} 35.7^{\prime \prime}$ & 29 \\
\hline San Blas & & & & 52 & $22^{\circ} 26^{\prime} 46.0^{\prime \prime}$ & $105^{\circ} 24^{\prime} 36.9^{\prime \prime}$ & 27 \\
\hline 24 & $21^{\circ} 40^{\prime} 55.4^{\prime \prime}$ & $105^{\circ} 21^{\prime} 06.5^{\prime \prime}$ & 13 & 53 & $22^{\circ} 26^{\prime} 46.4^{\prime \prime}$ & $105^{\circ} 24^{\prime} 37.1^{\prime \prime}$ & 27 \\
\hline 25 & $21^{\circ} 32^{\prime} 05.6^{\prime \prime}$ & $105^{\circ} 07^{\prime} 00.6^{\prime \prime}$ & 319 & 54 & $22^{\circ} 26^{\prime} 46.4^{\prime \prime}$ & $105^{\circ} 24^{\prime} 37.7^{\prime \prime}$ & 24 \\
\hline 26 & $21^{\circ} 38^{\prime} 55.9^{\prime \prime}$ & $105^{\circ} 06^{\prime} 46.8^{\prime \prime}$ & 50 & Tuxpan & & & \\
\hline 27 & $21^{\circ} 38^{\prime} 58.5^{\prime \prime}$ & $105^{\circ} 06^{\prime} 55.4^{\prime \prime}$ & 79 & 55 & $21^{\circ} 56^{\prime} 42.3^{\prime \prime}$ & $105^{\circ} 18^{\prime} 28.5^{\prime \prime}$ & 10 \\
\hline 28 & $21^{\circ} 39^{\prime} 02.8^{\prime \prime}$ & $105^{\circ} 07^{\prime} 01.4^{\prime \prime}$ & 103 & & & & \\
\hline
\end{tabular}

Cuadro 2. Valores promedio mínimos y máximos por municipio de las variables de hoja en 55 árboles de chicozapote evaluados en el estado de Nayarit.

\begin{tabular}{|c|c|c|c|c|c|c|}
\hline \multirow[t]{2}{*}{ Municipio } & \multicolumn{2}{|c|}{$\begin{array}{c}\text { Longitud de la hoja } \\
\qquad(\mathrm{mm})\end{array}$} & \multicolumn{2}{|c|}{$\begin{array}{l}\text { Ancho de la hoja } \\
(\mathbf{m m})\end{array}$} & \multicolumn{2}{|c|}{$\begin{array}{c}\text { Longitud de peciolo } \\
(\mathrm{mm})\end{array}$} \\
\hline & Mínimo & Máximo & Mínimo & Máximo & Mínimo & Máximo \\
\hline Ahuacatlán & 70.53 & 73.67 & 29.78 & 34.63 & 11.43 & 17.30 \\
\hline Amatlán de Cañas & 42.61 & 106.90 & 15.68 & 37.86 & 8.25 & 25.81 \\
\hline San Blas & 44.69 & 109.10 & 15.63 & 43.65 & 6.95 & 21.77 \\
\hline Santiago Ixcuintla & 42.08 & 115.57 & 14.73 & 42.80 & 7.64 & 28.73 \\
\hline Tecuala & 40.16 & 101.44 & 14.47 & 42.28 & 8.39 & 19.65 \\
\hline Tuxpan & & 63.38 & & 23.24 & & 11.51 \\
\hline
\end{tabular}


Cuadro 3. Valores promedio mínimos y máximos por municipio de las variables de peso de fruto en 55 árboles de chicozapote caracterizados en el estado de Nayarit.

\begin{tabular}{lcccccccc}
\hline \multicolumn{1}{c}{ Municipio } & \multicolumn{2}{c}{$\begin{array}{c}\text { Peso del fruto } \\
(\mathbf{g})\end{array}$} & \multicolumn{2}{c}{$\begin{array}{c}\text { Peso de la pulpa } \\
(\mathbf{g})\end{array}$} & \multicolumn{2}{c}{$\begin{array}{c}\text { Peso de cáscara } \\
(\mathbf{g})\end{array}$} & \multicolumn{2}{c}{$\begin{array}{c}\text { Peso de semilla } \\
(\mathbf{g})\end{array}$} \\
\hline & Min. $^{\mathbf{z}}$ & $\mathbf{M a x}^{\mathbf{x}}$ & $\mathbf{M i n}$. & \multicolumn{1}{c}{ Max. } & Min. & Max. & Min. & Max. \\
Ahuacatlán & 22.73 & 74.80 & 16.93 & 66.80 & 3.80 & 5.83 & 0.79 & 3.22 \\
Amatlán de Cañas & 28.50 & 179.00 & 22.50 & 155.67 & 4.45 & 19.20 & 0.00 & 4.94 \\
San Blas & 13.50 & 218.40 & 10.80 & 144.50 & 2.65 & 44.45 & 0.00 & 3.67 \\
Santiago Ixcuintla & 34.76 & 131.20 & 27.75 & 111.15 & 5.20 & 21.20 & 0.03 & 2.27 \\
Tecuala & 48.05 & 109.75 & 39.45 & 97.00 & 5.15 & 11.20 & 1.06 & 4.52 \\
Tuxpan & & 14.65 & & 11.75 & & 2.85 & & 0.45 \\
\hline
\end{tabular}

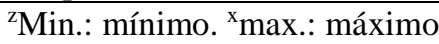

Cuadro 4. Valores promedio mínimos y máximos por municipio de los tamaños del fruto y grosor de la cáscara en 55 árboles de chicozapote caracterizados en el estado de Nayarit.

\begin{tabular}{|c|c|c|c|c|c|c|}
\hline \multirow[t]{2}{*}{ Municipio } & \multicolumn{2}{|c|}{$\begin{array}{c}\text { Diámetro longitudinal } \\
\text { del fruto } \\
(\mathbf{m m})\end{array}$} & \multicolumn{2}{|c|}{$\begin{array}{c}\text { Diámetro ecuatorial } \\
\text { del fruto } \\
(\mathbf{m m})\end{array}$} & \multicolumn{2}{|c|}{$\begin{array}{c}\text { Grosor de la } \\
\text { cáscara } \\
(\mathbf{m m})\end{array}$} \\
\hline & Min. $^{z}$ & Max. $^{x}$ & Min. & Max. & Min. & Max. \\
\hline Ahuacatlán & 39.56 & 53.04 & 35.83 & 54.39 & 0.20 & 0.38 \\
\hline Amatlán de Cañas & 37.80 & 65.99 & 36.37 & 67.08 & 0.29 & 0.72 \\
\hline San Blas & 34.22 & 89.12 & 31.48 & 68.12 & 0.15 & 1.32 \\
\hline Santiago Ixcuintla & 40.08 & 76.78 & 38.02 & 60.53 & 0.22 & 0.73 \\
\hline Tecuala & 40.62 & 63.14 & 45.67 & 63.37 & 0.21 & 0.36 \\
\hline Tuxpan & & 36.72 & & 32.60 & & 0.19 \\
\hline
\end{tabular}

${ }^{z}$ Min. : mínimo. ${ }^{\mathrm{x}} \max$ : : máximo

La recolecta 33 de Amatlán de Cañas destacó no sólo por el peso bajo, sino por el tamaño pequeño, diámetro longitudinal y ecuatorial del fruto de 34.47 y $25.87 \mathrm{~mm}$, respectivamente; la recolecta 2 sobresalió por el peso y grosor de la cáscara $(44.45 \mathrm{~g} \mathrm{y} 1.32 \mathrm{~mm}$ ); el menor grosor lo presentó la $4(0.15 \mathrm{~mm})$, otras tres recolectas presentaron valores medios, sin embargo, la mayoría se encontró por debajo de $0.52 \mathrm{~mm}$. La recolecta que presentó el mayor peso de semilla fue la 17 (4.94 g). 

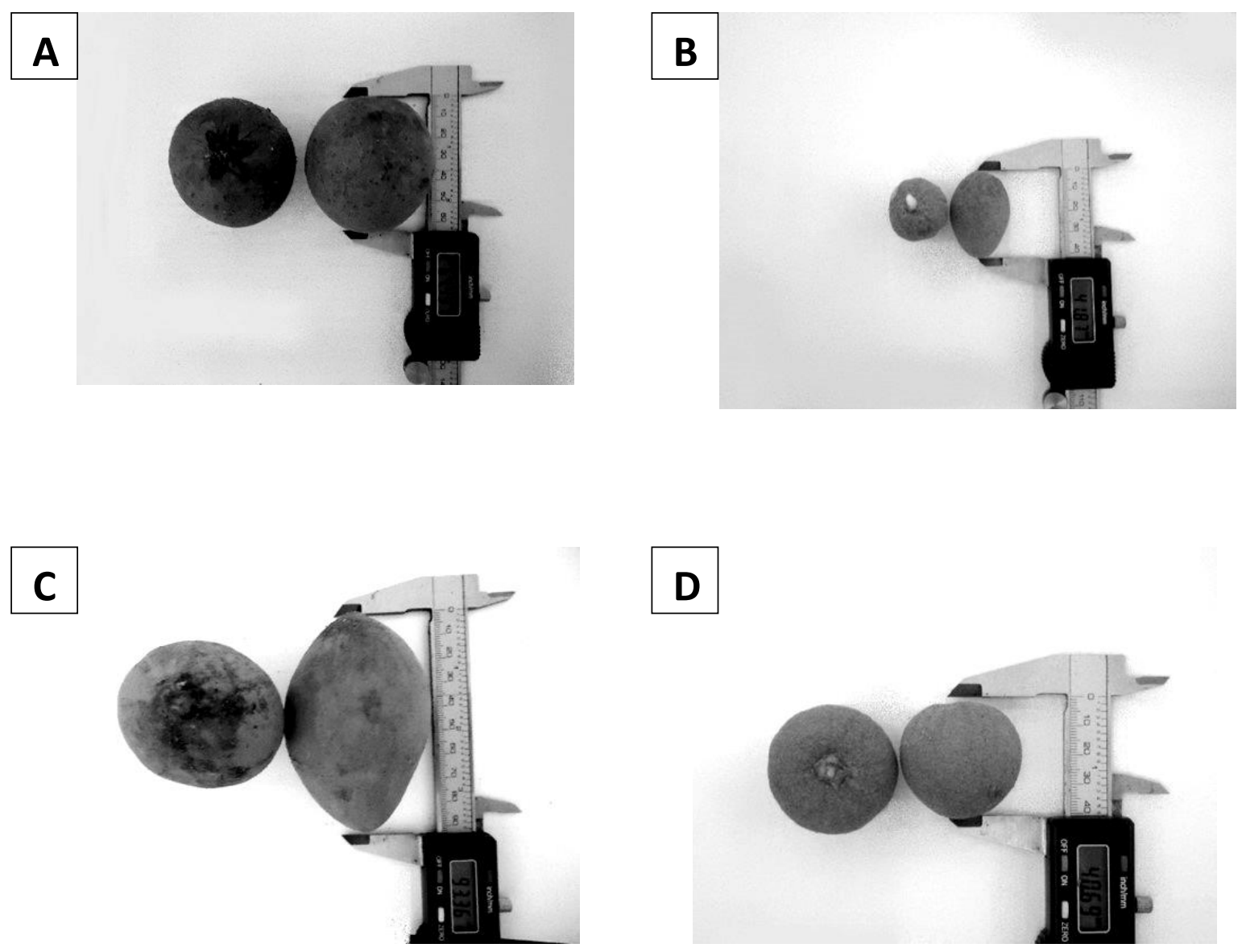

Fig. 1. Diversidad de forma y tamaño en frutos de chicozapote recolectados en Nayarit: fruto de forma redonda (A); fruto de forma alargada y tamaño pequeño $(41.87 \mathrm{~mm})(B)$; fruto de forma alargada de tamaño grande $(93.36 \mathrm{~mm})$ (C); y, fruto con diámetro ecuatorial mayor que el diámetro polar (D).
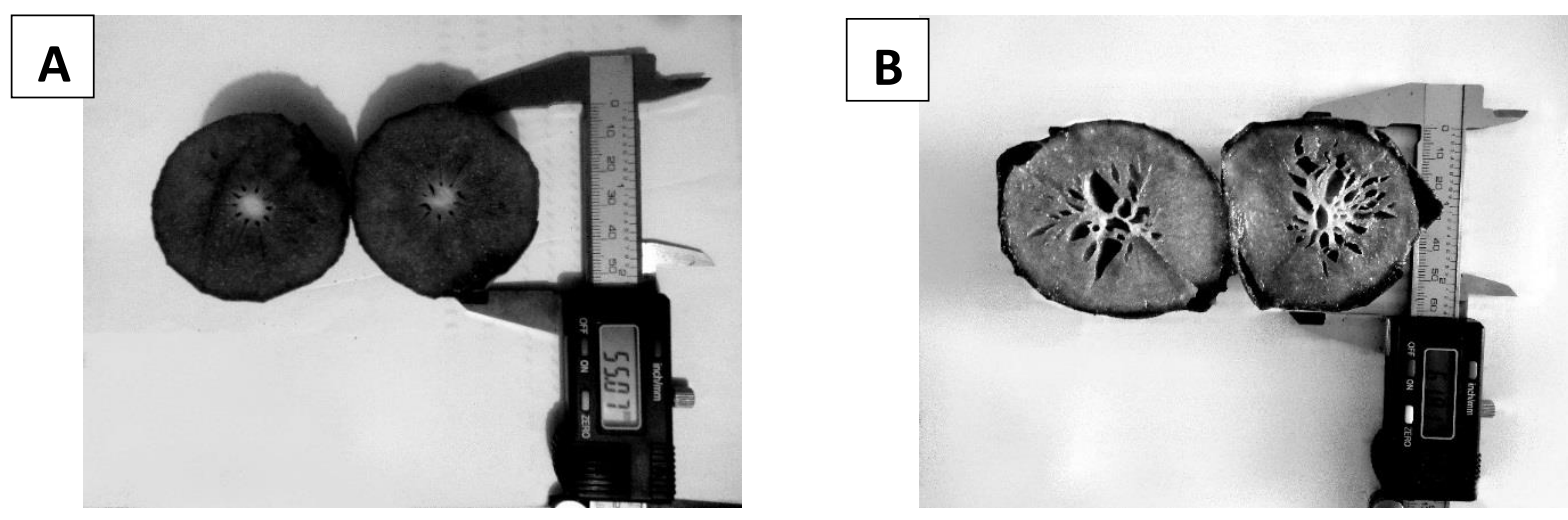

Fig. 2. Frutos de chicozapote recolectados en Nayarit: sin semillas (A); y con semillas (B). 
El número de semillas por fruto se presenta en el cuadro 5. El mayor número lo presentó la recolecta 23 (6.70 semillas por fruto), tres recolectas presentaron frutos sin semilla (fig. 2), 22 presentaron valores menores a uno, lo que significa que pocos frutos de la muestra tenían semillas; y sólo 22 recolectas tuvieron frutos que siempre presentaron semillas. Los datos de largo, ancho y grosor de la semilla se presentan en el cuadro 5.

Cuadro 5. Valores promedio mínimos y máximos por municipio de las variables evaluadas para la caracterización de semillas de 55 recolectas de chicozapote en el estado de Nayarit.

\begin{tabular}{|c|c|c|c|c|c|c|c|c|}
\hline \multirow[t]{2}{*}{ Municipio } & \multicolumn{2}{|c|}{$\begin{array}{l}\text { Número de } \\
\text { semillas }\end{array}$} & \multicolumn{2}{|c|}{$\begin{array}{l}\text { Largo } \\
(\mathbf{m m})\end{array}$} & \multicolumn{2}{|c|}{$\begin{array}{c}\text { Ancho } \\
\text { (mm) }\end{array}$} & \multicolumn{2}{|c|}{$\begin{array}{c}\text { Grosor } \\
(\mathbf{m m})\end{array}$} \\
\hline & Min. & Max. & Min. & Max. & Min. & Max. & Min. & Max. \\
\hline Ahuacatlán & 1.50 & 5.00 & 12.36 & 19.80 & 6.43 & 10.35 & 3.25 & 4.98 \\
\hline Amatlán de Cañas & 0.40 & 3.47 & 2.80 & 15.12 & 1.41 & 7.19 & 0.81 & 4.00 \\
\hline Compostela & 0.00 & 5.28 & 0.00 & 24.35 & 0.00 & 10.14 & 0.00 & 5.78 \\
\hline San Blas & 0.00 & 4.20 & 0.00 & 23.11 & 0.00 & 12.24 & 0.00 & 5.54 \\
\hline Santiago & 0.05 & 3.60 & 0.48 & 17.09 & 0.24 & 8.66 & 0.14 & 4.53 \\
\hline Tecuala & 1.35 & 6.70 & 10.89 & 23.79 & 6.79 & 11.70 & 3.51 & 5.83 \\
\hline
\end{tabular}

\section{Análisis de componentes principales}

Los datos sobre caracterización de la semilla no se utilizaron para el análisis de componentes principales, debido a que algunos genotipos no presentaban semilla y no se ajustaban a los supuestos del análisis (media 1 y varianza 0), y debido a la colinealidad que presentaban las variables peso de semilla y número de semillas que se correlacionaron en $94.13 \%$, no se utilizó la variable número de semillas, para el análisis.

El resultado del análisis de componentes principales (CP) se muestra en el cuadro 6. Los tres primeros componentes explicaron $79.13 \%$ de la variabilidad morfológica total. El CP1 explicó el $47 \%$ de la variabilidad, y las variables que contribuyeron fueron diámetro ecuatorial y longitudinal del fruto y peso del fruto y peso de la pulpa. El CP2 explicó el $19.93 \%$ de la variabilidad y está determinado por el ancho de la hoja; por último, el CP3 explicó el $11.56 \%$ de la variabilidad total y el descriptor que lo determina es el peso de la semilla.

En la figura 3 se muestra la distribución de la variabilidad de árboles de chicozapote en función de los dos primeros componentes principales. Se observa que las recolectas del 3 al 11 se agrupan en el cuadrante izquierdo, estas provienen de los municipios de Ahuacatlán y Amatlán de Cañas sitios geográficamente cercanos lo que sugiere un origen común, sin embargo, no se encuentran todas las recolectas provenientes de ésos municipios y también se encuentran recolectas de San Blas. Las demás recolectas de los municipios están mezcladas y distribuidas en el diagrama.

\section{Dendrograma de similitud}

Al realizar el análisis de conglomerados jerárquicos se identificaron cinco grupos a una distancia euclidiana de 0.08 (fig. 4). 
Cuadro 6. Vectores propios, varianza explicada y varianza acumulada en los tres primeros componentes principales en 55 recolectas de chicozapote en Nayarit.

\begin{tabular}{lccc}
\hline \multirow{2}{*}{ Descriptor } & \multicolumn{3}{c}{ Componentes principales /Vectores propios } \\
\cline { 2 - 4 } & CP1 & CP2 & CP3 \\
\hline Diámetro longitudinal del fruto & 0.3368 & 0.0539 & -0.2422 \\
Diámetro ecuatorial del fruto & 0.3984 & -0.1995 & 0.0427 \\
Peso del fruto & 0.4142 & -0.2388 & -0.0345 \\
Peso de la pulpa & 0.4134 & -0.2092 & -0.0201 \\
Peso de la cáscara & 0.3728 & -0.2537 & -0.1650 \\
Peso de la semilla & 0.9895 & -0.1776 & 0.8966 \\
Grosor de la cáscara & 0.0964 & -0.2918 & 0.0862 \\
Longitud de la hoja & 0.3079 & 0.4700 & 0.0968 \\
Ancho de la hoja & 0.2359 & 0.5166 & 0.1563 \\
Longitud del peciolo & 0.2769 & 0.4342 & 0.0711 \\
\hline Valor propio & 4.7639 & 1.9934 & 1.5567 \\
Varianza explicada & 0.4764 & 0.1993 & 0.1156 \\
Varianza acumulada & 0.4764 & 0.6767 & 0.7913 \\
\hline
\end{tabular}

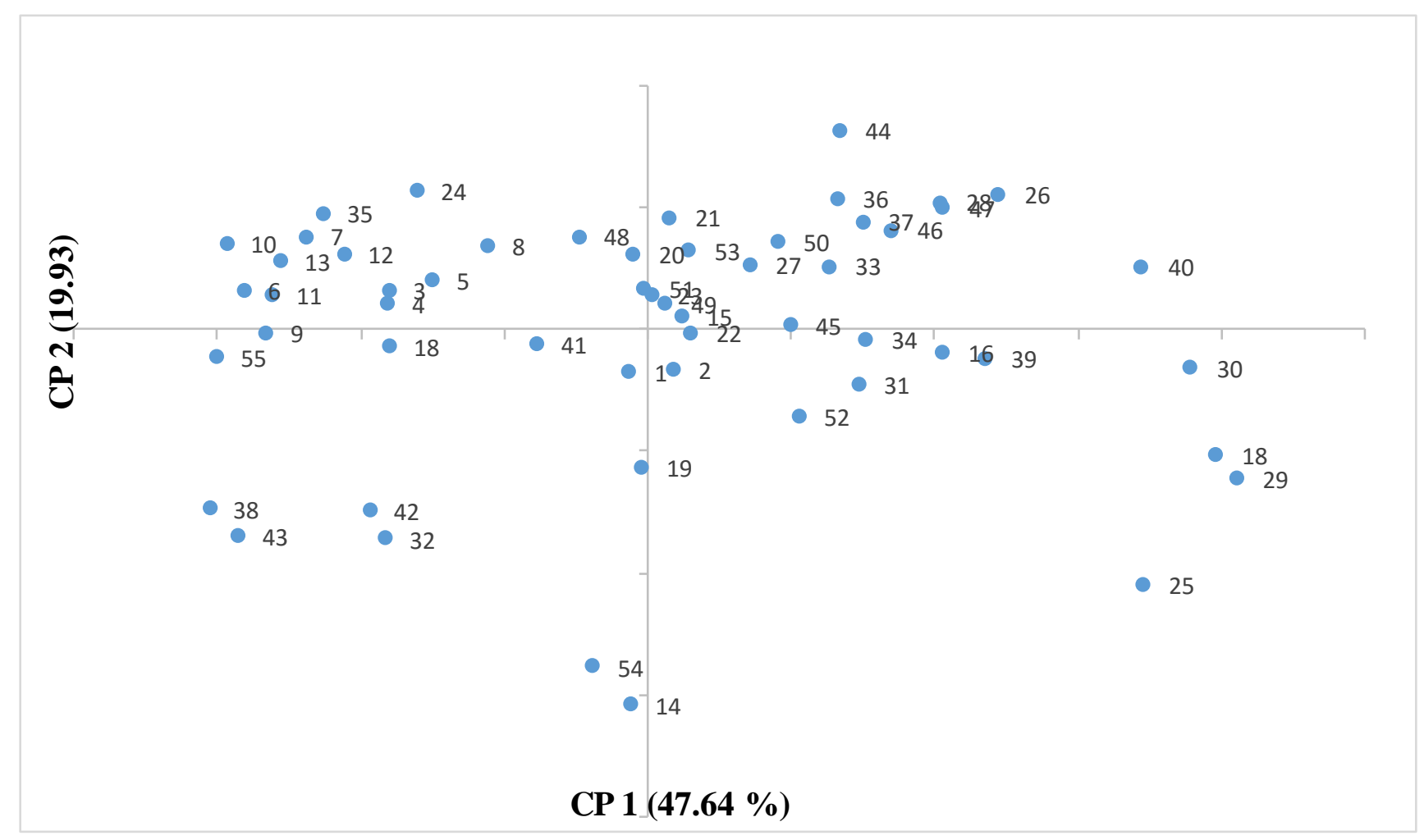

Fig. 3. Distribución gráfica de la variabilidad de árboles de chicozapote en función de los dos primeros componentes principales: las recolectas de los diferentes municipios se encuentran mezcladas. 


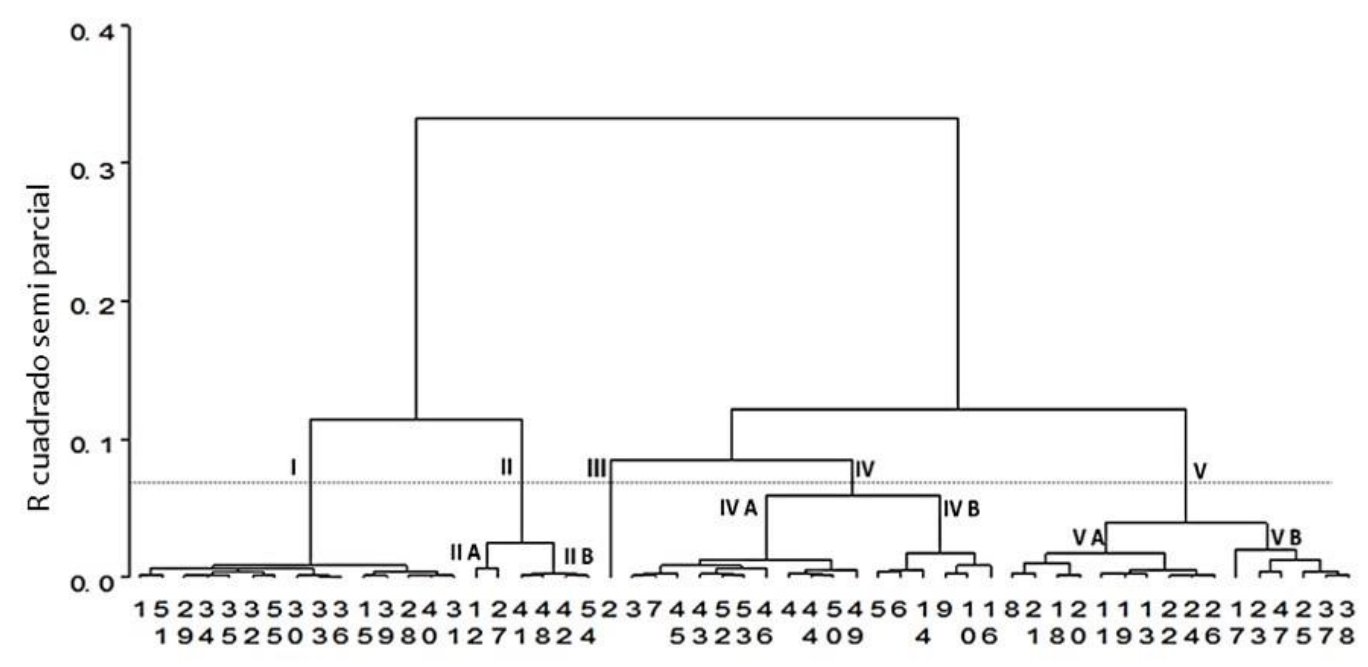

Nombre de la observación o conglomerado

Fig. 4. Dendrograma obtenido del análisis de agrupamiento con el método de análisis de conglomerados jerárquicos mediante UPGMA de 55 recolectas de chicozapote en Nayarit: la variabilidad mostrada en las recolectas permitió la formación de subgrupos. ${ }^{2}$ Número de grupo.

El grupo I estuvo conformado por 16 recolectas con frutos de peso promedio de fruto y pulpa alto $(89.55$ y $72.77 \mathrm{~g})$; los frutos eran de ancho similar al largo (redondeados, relación diámetro polar/ecuatorial de 1.09) y hojas grandes $(86.26$ y $32.95 \mathrm{~mm}$ de largo y ancho, respectivamente). En el grupo II se agruparon 6 recolectas con frutos de peso bajo (50.46 g) y hojas de tamaño mediano (70.61 x $27.78 \mathrm{~mm})$; que, a una distancia de 0.26 formó dos subgrupos, uno con forma de fruto alargada (relación diámetro polar/ecuatorial del fruto de 1.52) y otro con frutos redondeados, ligeramente achatados en los polos (relación diámetro polar/ecuatorial 0.96). El grupo III estuvo conformado por una sola recolecta, con frutos de forma redondeada (relación diámetro polar/ ecuatorial de 98), peso promedio total y de pulpa medio (74.80 y $66.80 \mathrm{~g}$ ); hoja de tamaño medio $(73.67$ x $30.64 \mathrm{~mm})$; pero peso de semilla alto (3.22 g). El grupo IV estuvo conformado por 17 recolectas de frutos ligeramente alargados (relación diámetro polar/ecuatorial de 1.19) y peso promedio medio (56.01 y $47.27 \mathrm{~g}$ ); se formaron dos subgrupos que se diferenciaron por el peso de las semillas, que fue de $1.56 \mathrm{~g}$ en el primero (IV A) y de $0.60 \mathrm{~g}$ en el segundo (IV B). Por último, el grupo V, estuvo conformado por 15 recolectas de forma ligeramente alargada (relación diámetro polar/ecuatorial de 1.25), y hojas grandes $(82.52 \times 31.22 \mathrm{~mm})$.

\section{DISCUSIÓN}

La fecha de cosecha de los frutos de chicozapote en Nayarit fue de marzo a septiembre, mientras que (Villegas, 2012) presenta las fechas de cosecha para el sureste de México de diciembre a marzo, dependiendo del cultivar. Por lo anterior los materiales ubicados en Nayarit pueden ser utilizados para obtener cosecha de marzo a septiembre y surtir al mercado con chicozapote durante todo el año. Además, los propietarios de la recolecta 11 indicaron que los árboles presentaban dos cosechas al año, una en abril y otra en diciembre.

La vida postcosecha de los frutos, recolectados en Nayarit, desde madurez fisiológica (fecha de cosecha) a madurez de consumo (fecha de caracterización) fue desde los 4 a los 29 días. Se 
identificaron materiales con vida de anaquel larga, como las recolectas 1, 6, 20, 29, 35, 36, 39 y 51 que fue de 20 a 29 días. Esta característica es importante porque la comercialización del chicozapote es limitada debido a que es un fruto altamente perecedero (Bolívar, Saucedo y Sauri, 2011).

De acuerdo a Vázquez et al., 1999 las hojas de chicozapote miden entre 5.5 a $18 \mathrm{~cm}$ de largo y 2 a $7 \mathrm{~cm}$ de ancho. Las hojas recolectadas en Nayarit estuvieron dentro de los valores mediobajos de esta referencia y similares a los reportados por Aguilar, Corona, Juárez y Villanueva (2018) para los estados de Veracruz y Morelos.

Considerando los valores presentados en las recolectas, se encontró que el largo y ancho del fruto (cuadro 2, figura 1) está fuera del rango reportado por Vázquez et al. (1999), quienes indicaron que los frutos de chicozapote miden entre 5 y $10 \mathrm{~cm}$ de largo, ya que en el Estado se encontraron frutos de tamaño muy pequeño.

En cuanto al grosor de cáscara los valores difieren de lo reportado por Gazel (2002), que presenta medias de grosor de cáscara de 0.8 a $1.2 \mathrm{~mm}$. La importancia del grosor de la cáscara en que puede tener influencia en la resistencia mecánica al manejo en cosecha y poscosecha del fruto como lo reportaron para plátano (Osuna, Vázquez y Pérez, 2008). La recolecta con mayor grosor de cáscara, también presentó el mayor peso de cáscara (44.45 g), sin embargo, debe considerarse que este valor también es afectado por el tamaño del fruto.

El peso de la semilla en frutos de Nayarit es similar a los valores altos reportados por (Gazel, 2002) de 3.5, 3.8 y $4.8 \mathrm{~g}$ para los tres grupos que se formaron, sin embargo, este autor no reporta materiales sin semilla; mientras que, Aguilar, Corona, Juárez \& Villanueva (2018) reportan materiales con contenido promedio de semillas por fruto, cercanos a cero, para la región de Azapan-Agua Caliente en el estado de Veracruz. En este trabajo, se encontraron materiales con gran cantidad de semillas y sin semillas. La producción de fruto sin semilla se considera un atributo para la exportación (Villegas, 2012). Aunque para consumo de frutos se buscan genotipos sin semillas en la actualidad existe interés en el polímero del mucílago de semillas de chicozapote que aún no ha sido comercialmente explotado (Otari, Patil, Ghosh y Pawar, 2014; Singh y Bothara, 2014), además del interés que existe en la capacidad bactericida de amplio espectro encontrado en extractos acuosos de semillas de chicozapote (Kothari y Seshadri, 2010).

En cuanto al tamaño de la semilla, de acuerdo a Vázquez et al. (1999) las semillas de chicozapote miden entre 16 a $23 \mathrm{~mm}$ de largo por 8 a $16 \mathrm{~mm}$ de ancho. En este caso se encontraron 36 recolectas que presentaron semillas con longitud menor a $8 \mathrm{~mm}$; el tamaño menor estaba relacionado a semillas vanas. La presencia de semillas vanas puede indicar aborto del embrión, a diferencia de los frutos sin semillas que son partenocárpicos.

Se ha reportado que el chicozapote presenta autoincompatibilidad, sin embargo, Kishore, Samant, Singh y Behera (2017) estudiaron las estrategias reproductivas en chicozapote y determinaron que las flores presentan hercogamia, pobre apertura floral y número de polinizadores pequeño, pero estos autores consideran que el chicozapote es autocompatible ya que obtuvieron más del $25 \%$ de amarre de fruto en flores autopolinizadas. No obstante, la incompatibilidad gametofítica produce frutos partenocárpicos (Santiaguillo, Cervantes, Peña, Molina y Sahagún, 2017) y el grado de incompatibilidad puede variar entre genotipos y dependiendo de las condiciones ambientales (Grijalva et al., 2015). Debido a lo anterior, los árboles de las recolectas con frutos partenocárpicos pueden ser utilizados para generar más información sobre autoincompatibilidad en chicozapote.

No fue posible distinguir en los grupos formados en el dendograma, la región de origen de las recolectas. Aguilar, Torres, Corona, Juárez \& Villanueva (2018) al realizar el análisis de conglomerados lograron distinguir los árboles criollos del estado de Morelos en un grupo 
debido a que no se asemejaban al resto de los grupos de la región centro del estado de Veracruz, y consideraron que tenían un origen diferente. El que no sea posible identificar los grupos con las áreas geográficas en este trabajo puede ser debido a que el chicozapote es una especie introducida $\mathrm{y}$, probablemente, la diversidad este más relacionada con el origen de las introducciones; o a que las introducciones sean de origen relativamente reciente por lo que no han desarrollado poblaciones con características distintivas.

\section{CONCLUSIONES}

Existe variabilidad morfológica entre los materiales de chicozapote presentes en Nayarit. Algunos de los materiales presentan características adecuadas para producción de fruta: vida postcosecha larga, no poseer semillas y cáscara gruesa que pueden ser evaluados por sus características organolépticas. Los árboles productores de frutos partenocárpicos tienen el potencial de ser utilizados para estudios de biología reproductiva de chicozapote.

\section{LITERATURA CITADA}

Aguilar R., V. H., Corona T., T., Juárez G., P., Villanueva J., J. A. (2018) Caracterización morfológica del chicozapote (Manilkara zapota) del centro de Veracruz y Morelos, México: base para su mejoramiento. Producción Agropecuaria y Desarrollo Sostenible. 7 https://doi.org/10.5377/payds.v7i0.8411.

Aldrete, T. M. \& Ramírez, G. (2005). Chicle natural: Producto que conserva la selva tropical del Gran Peten. La Riqueza de Los Árboles Mexicanos, Más Allá de La Madera: Experiencia de Comunidades Rurales, pp. 92-99. Retrieved from http://www.cifor.org/publications/pdf_files/Books/BLopez0501S0.pdf

Azurdia, C. (2006). Tres Especies de Zapote en América Tropical (Pouteria campechiana, P. sapota y P. viridis). Southampton Centre for Underutilised Crops, Universidad de Southampton, Southampton, UK. Retrieved from https://core.ac.uk/download/pdf/27770.pdf?repositoryId=34

Bautista R., B., Arévalo G., M. de L., Veloz S., C.\& Martínez D., M. T. (2005). Proceso de maduración de frutos de chicozapote [Manilkara sapota (L.) P. Royen] tipo Fino. Rev. Chapingo Ser. Hortic., 11, 387-391.

Bolívar F., N., Saucedo V., C. \& Sauri D., E. (2011). Respiración y parámetros relacionados durante la maduración del chicozapote cosechado en la Península de Yucatán. Rev. Bras. Frutic., Jaboticabal - SP, Volume Esp, 261-266. https://doi.org/http://dx.doi.org/10.1590/S0100-29452011000500032.

Brito, E. S. De \& Narain, N. (2002). Physical and chemical characteristics of sapota fruit at difeferent stages of maduration. Pesq. Agropec. Bras., Brasília, 37(4), 567-572. https://doi.org/10.1590/S0100-204X2002000400020.

CONAP (Comisión Nacional de Áreas Naturales Protegidas). (2016). Inicia en Campeche temporada de producción de chicle y miel. Retrieved from http://www.gob.mx.

Gazel, A. B. (2002). Caracterizacion de plantas de chicozapote (Manilkara zapota (L.) P. van Royen) de la colección del CATIE,mediante el uso de análisis multivariado. Rev. Bras. Frutic., Jaboticabal -, 24(3), 727-730. Retrieved from http://www.scielo.br/pdf/rbf/v24n3/15125.pdf

González H., D., García P., E. \& Guntin M., P. (2012). Caracterización genética de Manilkara zapota de Veracruz, México con marcadores moleculares. Agrociencia, 46(7), 663675. Retrieved from http://www.scielo.org.mx/scielo.php? script=sci_arttext\&pid=S1405-31952012000700003\&lng=es\&nrm=iso

Grijalva C., R. L., Macías D., R., López C., A., Martínez D., G., Nuñez R., F. \& Robles C., F. (2015). Supplemental pollination with different sources of pollen in olive (Olea europaea) 'Manzanilla' under hot and arid environment. ARRB, 7(6), 363-369. 


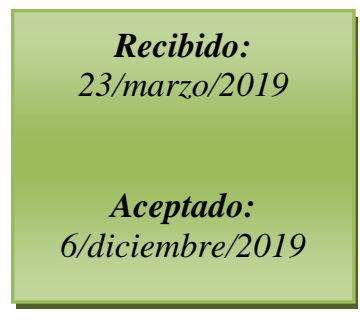

https://doi.org/10.9734/arrb/2015/19297

Heaton, H. J., Whitkus, R., \& Gomez P., A. (2003). Extreme ecological and phenotypic differences in the tropical tree chicozapote (Manilkara zapota (L.) P. Royen) are not matched by genetic divergence: a random amplified polymorphic DNA (RAPD) analysis. Mol Ecol, 8(4), 627-632. https://doi.org/10.1046/j.1365-294x.1999.00616.x

Kishore, K., Samant, D., Singh, H. S. \& Behera, S. (2017). Studies on the reproductive strategies of sapota (Manilkara zapota). J. Environ. Biol., 38(3), 361-366. https://doi.org/10.22438/jeb/38/3/ms-264

Kothari, V., \& Seshadri, S. (2010). In vitro antibacterial activity in seed extracts of Manilkara zapota, Anona squamosa, and Tamarindus indica. Biol Res, 43, 165-168.

Moo H., V. M., Estrada L., R., Estrada M., I. A., Cuevas G., L. F. \& Sauri D., E. (2013). Ripening of sapodilla fruits (Manilkara zapota [ L .] P . Royen) treated with 1methylcyclopropene after refrigeration. Afr. J. Plant Sci., 7, 561-570. https://doi.org/10.5897/AJPS2013.1088

Osawaru, M. E., Ogwu, M. C. \& Aiwansoba, R. O. (2015). Hierarchical approaches to the analysis of genetic diversity in crop plants: A systematic overview. University of Mauritius Research Journal, 21, 1-36. Retrieved from https://www.ajol.info/index.php/umrj/article/view/122070/111548

Osuna G., J. A., Vázquez V., V. \& Pérez B., M. H. (2008). Caracterización poscosecha de cultivares de plátano para consumo en fresco. Rev. Chapingo Ser. Hortic., 14(2), 139145. Retrieved from http://www.scielo.org.mx/scielo.php? script=sci_arttext\&pid=S1027-152X2008000200006\&lng=es\&nrm=iso

Otari, S. V., Patil, R. M. Ghosh, S. J. \& Pawar, S. H. (2014). Green phytosynthesis of silver nanoparticles using aqueous extract of Manilkara zapota ( L .) seeds and its inhibitory action against Candida species. Materials Letters, 116, 367-369. https://doi.org/10.1016/j.matlet.2013.11.066

Paredes, M., Becerra, V., Condón, F., Germán, S. \& Barbieri, R. L. (2006). Utilzación de los recursos fitogenéticos. In: Estrategia en los recursos fitogenéticos para los países del cono sur (pp. 97-116). https://doi.org/10.1590/s0103-40142000000100005

Piñero, D., Caballero M., J., Cabrera T., D., Canteros, C. E. \& Casas, A. (2008). La diversidad genética como instrumento para la conservación y el aprovechamiento de la biodiversidad: estudios en especies mexicanas. In Capital natural de México, vol. I: Conocimiento actual de la biodiversidad. (pp. 437-494). Retrieved from https://s3.amazonaws.com/academia.edu.documents/45331837/La_diversidad_gentica _como_instrumento_p20160503-23169-

1jwy62d.pdf?AWSAccessKeyId=AKIAIWOWYYGZ2Y53UL3A\&Expires=1556773 669\&Signature=D1H\%2BfzuYIQRhJHVRoQqIuiwTIRM\%3D\&response-contentdisposition=inli

Santiaguillo H., J. F., Cervantes S., T., Peña L., A., Molina G., J. D. \& Sahagún C., J. (2005). Polinización controlada en tomate de cáscara (Physalis ixocarpa Brot.). Rev. Chapingo Ser. Hortic., 11(1), 67-71. https://doi.org/10.5154/r.rchsh.2003.10.064

Singh, S. \& Bothara, S. B. (2014). Manilkara zapota (Linn.) Seeds: A Potential Source of Natural Gum. ISRN Pharmaceutics, 2014, 1-10. https://doi.org/10.1155/2014/647174

Vázquez Y., C., Batis M., A. I., Alcocer S., M. I., Gual D., M. \& Sánchez D., C. (1999). Manilkara zapota. Arboles y arbustos nativos potencialmente valiosos para la restauración ecológica y la reforestación, pp. 239-242. Retrieved from http://www.conabio.gob.mx/conocimiento/info_especies/arboles/doctos/64sapot $4 \mathrm{~m} . \mathrm{pdf}$

Villegas, A. (2012). Conservación y aprovechamiento de los recursos fitogenéticos en las Sapotáceas. In M. G. S. M. (Coordindores) S. Espinoza Z., A. Villegas, M.C. Avendaño, O. López B., J. L. Moreno (Ed.), Zapote mamey y otras sapotáceas (pp. 39-68). Editorial Ambiente S.A. de C.V. 